\title{
Adipose Tissue Derived Stem Cells Secretome: Soluble Factors and Their Roles in Regenerative Medicine
}

\author{
António J. Salgado*,1, Rui L. Reis ${ }^{2,3}$, Nuno Sousa ${ }^{1}$ and Jeffrey M. Gimble*,4 \\ ${ }^{1}$ Life and Health Sciences Research Institute (ICVS), School of Health Sciences, University of Minho, Braga, Portugal; ${ }^{2} 3 B$ 's \\ Research Group - Biomaterials, Biodegradables and Biomimetics, University of Minho, Headquarters of the European Institute of \\ Excellence on Tissue Engineering and Regenerative Medicine, AvePark, Taipas, Guimarães, Portugal; ${ }^{3}$ IBB, Institute for Bioengi- \\ neering and Biotechnology, PT Associated Lab, Braga, Portugal; ${ }^{4}$ Stem Cell Biology Laboratory, Pennington Biomedical Research \\ Center, Louisiana State University System, Baton Rouge, LA 70808, USA
}

\begin{abstract}
Stem cells have been long looked at as possible therapeutic vehicles for different health related problems. Among the different existing stem cell populations, Adipose derived Stem Cells (ASCs) have been gathering attention in the last 10 years. When compared to other stem cells populations and sources, ASCs can be easily isolated while providing higher yields upon the processing of adipose tissue. Similar to other stem cell populations, it was initially thought that the main potential of ASCs for regenerative medicine approaches was intimately related to their differentiation capability. Although this is true, there has been an increasing body of literature describing the trophic effects of ASCs on the protection, survival and differentiation of a variety of endogenous cells/tissues. Moreover, they have also shown to possess an immunomodulatory character. This effect is closely related to the ASCs' secretome and the soluble factors found within it. Molecules such as hepatocyte growth factor (HGF), granulocyte and macrophage colony stimulating factors, interleukins (ILs) $6,7,8$ and 11 , tumor necrosis factor- $\alpha(\mathrm{TNF}-\alpha)$, vascular endothelial growth factor (VEGF), brain derived neurotrophic factor (BDNF), nerve growth factor (NGF), adipokines and others have been identified within the ASCs' secretome. Due to its importance regarding future applications for the field of regenerative medicine, we aim, in the present review, to make a comprehensive analysis of the literature relating to the ASCs' secretome and its relevance to the immune and central nervous system, vascularization and cardiac regeneration. The concluding section will highlight some of the major challenges that remain before ASCs can be used for future clinical applications.
\end{abstract}

Keywords: Adipose tissue derived stem cells, secretome, soluble factors, cell survival, cell differentiation, trophic support.

\section{INTRODUCTION}

Once considered to be a passive type of connective tissue storing excess energy as triglyceraldheydes, adipose tissue has now been established as an endocrine organ coupling (neuro)-endocrine and metabolic signaling [1]. Through the years there has been an increasing body of knowledge showing that the secretory products of this tissue regulate energy homeostasis, appetite/satiety, reproduction and insulin sensitivity. Simultaneously adipose tissue is also able to influence the neuroendocrine, endothelial, immunological, hematological, angiogenic and vascular functions in an endocrine, paracrine and autocrine manner [1,2]. Adipose-derived Stem or Stromal Cells (ASCs) within adipose depots are responsible for the regeneration of the tissue over time by replacing the $\sim 10 \%$ of mature adipocytes that turn over each year [3]. Similar to the broad function of the tissue from which they can be isolated, ASCs may have a larger spectrum of applications and functions than was initially thought. As with bone marrow-derived mesenchymal stem cells (BMSCs), the classical example of adult stem cell populations, it was believed that the major value of ASCs was mainly related to their differentiation capability towards different mature lineages. However recent evidence has shown that this paradigm may be somewhat limited. In recent years, it has been shown that the ASCs' secretome, consisting of the proteins and growth factors secreted into the extracellular milieu, have a beneficial impact in different organs/systems within the human body. The objective of this review is to provide a comprehensive analysis focused on the ASCs' secretome, namely its constitution and most relevant factors,

*Address correspondence to these authors at the Life and Health Sciences Research Institute (ICVS), School of Health Sciences, University of Minho, Braga, Portugal; Tel: +351 2536049 47; Fax: +351 2536048 20;

E-mail: asalgado@ecsaude.uminho.pt

Stem Cell Laboratory, Pennington Biomedical Research Center, Louisiana State University System, Baton Rouge, LA 70808, USA;

E-mail: jeffrey.gimble@pbrc.edu and its actions on different organs and systems in different animal models. This review will start by giving a brief synopsis of ASCs' basic biology followed by a section dedicated to their secretome, concluding with a summary of its potential impact in different models of injury and disease.

\section{SYNOPSIS OF ASCs BASIC BIOLOGY}

The existence of a population of multipotent stem cells within the adipose tissue arose after observations from pathological events within this tissue, such as progressive osseous heteroplasia and obesity $[4,5]$. The first is characterized by ectopic bone formation within the subcutaneous adipose layer of the skin [4,6-8]. Closer observations to these ectopic formations also revealed the presence of osteoblasts and chondrocytes, in addition to adipocytes [6], which were a clear indication of the presence of a multipotent stem cell population within the adipose tissue. In addition to this, obesity further reinforced this hypothesis [4,5]. In vivo models of adipogenesis suggest that the adipocyte is in fact a terminally differentiated cell, with a limited capacity of proliferation and a turnover rate of around 6-15 months [9-11]. With this background, it was plausible to accept the existence of a population of adipose-derived stem cells that had pre-adipocyte function and were responsible for the replacement of terminal adipocytes throughout the life of an adult individual but were also capable of alternative lineage differentiation [4].

While Bjorntorp, Hauner, Van, and their colleagues first isolated pre-adipocytes from rodent and human adipose depots over three decades ago [12-14] the nomenclature relating to ASCs and their isolation appeared for the first time almost ten years ago [1517]. Since then, ASCs have been reported to be found within the different white and brown adipose tissue depots (WAT or BAT) present in the body [18]. The simple surgical procedure, the easy and repeatable access to adipose tissue, and the uncomplicatedbased isolation procedures make this source quite attractive for both research and/or biomedical applications [1] when compared, for instance, to BMSCs. Current methods for its isolation are generally 
based on enzymatic digestion of the adipose tissue followed by centrifugation to isolate the stromal/vascular fraction from primary adipocytes [5,19-21]. Upon isolation this stromal/vascular fraction will adhere to tissue culture plastic, while the hematopoietic fraction and other contaminating cells can be depleted by different techniques.

After in vitro expansion, ASCs acquire a fibroblast like morphology, similar to the one observed for BMSCs [16], which are still considered as the gold standard within the field. Furthermore, ASCs also express a series of cell-specific proteins and CD markers phenotypically similar to BMSCs [19,22-24]. Further details on these topics can be found elsewhere $[19,22-24]$ and are summarized in Table 1 regarding adhesion and receptor molecules, surface enzymes, extracellular matrix proteins and glycoproteins, skeletal proteins, complement regulatory proteins.

Further research carried out by different groups also revealed the in vitro differentiation of these cells into osteogenic [16,22,25], chondrogenic [26,27], myogenic [28], neuronal-like [16,29,30], cardiomyocyte [31,32], epithelial [33,34] and endothelial lineages $[35,36]$.

\section{ASCs SECRETOME - BASICS AND POSSIBLE APPLI- CATIONS}

The secretome of ASCs has the potential to be a powerful tool in future approaches in developing cell/tissue based therapeutics for regenerative medicine. In fact, as it will be discussed later on and as shown in Table 2, there are numerous examples of the to beneficial effects that the ASC's secretome exerts in the central nervous systems (CNS), immune system, heart, muscle and even in general cell survival. There has been an extensive literature [37-42] dealing with the secretory profile of pre-adipocytes, ASCs, or adipose tissue as determined by Enzyme Linked Immunoabsorbent Assay (ELISA) or related techniques. Nevertheless, the profile of the secretory products of ASCs has only recently begun to be characterized at the mass spectrometry level. Although there are a number of papers dedicated to ASCs proteomic characterization [43-46], most of them are actually focused on the proteome of cell lysates rather than the characterization of the secretome itself. A recent study characterized the ASCs secretome in the context of differentiation towards the adipogenic lineage [47]. The results were obtained after 2D gel electrophoresis and tandem mass spectrometry of CM obtained after 16 hours of culture from undifferentiated or day 9 adipocyte differentiated P2 human ASCs. The latter allowed the identification of 101 individual proteins in the CM. Regarding the subcellular location and functional categories of the secretome the following results were reported:

\section{Subcellular Location}

The proteins in the CM displayed the following characteristics: $41 \%$ had cytoplasmic associations, $15 \%$ were known secretory factors, $11 \%$ were nuclear derived, $4 \%$ were from the endoplasmatic reticulum, 3\% were attributed to the extracellular matrix (ECM), $4 \%$ were mitochondrial and $22 \%$ were not associated with a specific subcellular location.

\section{Function}

Regarding the function of the identified proteins $30 \%$ were related to cell metabolism, $7 \%$ were cytoskeletal, $8 \%$ were attributed to cell signaling, $7 \%$ to protein inhibitors, another $7 \%$ to protein degradation, $6 \%$ to protein processing, $3 \%$ were chaperones, $16 \%$ related to DNA repair, extracellular matrix, iron storage, GDPbinding protein and mitosis, and $6 \%$ did not have an attributed function.

This "contamination" with intracellular proteins could be due to leakage or, alternatively, to contamination related to cell death. In addition, other mechanisms such as exosome secretion cannot also be excluded. The presence of such cytosolic "contamination" is a potential artefact that can occur when working with either cell culture or tissue extract samples. Nevertheless, the fact that $29 \%$ of the 101 identified proteins contained a predicted signal peptide consis-

Table 1. Surface Proteins Positively Identified in Adipose-Derived Adult Stem Cells [Adapted from 19 and 24]

\begin{tabular}{|c|c|c|}
\hline Protein Category & Protein Name & CD Number \\
\hline Adhesion molecules & $\begin{array}{c}\text { Tetraspan Protein } \\
\text { Integrins b1 } \\
\text { Integrins a4 } \\
\text { Intercellular Adhesion Molecule 1 } \\
\text { Endoglin } \\
\text { Vascular Cell Adhesion Molecule } \\
\text { Activated Lymphocyte Cell Adhesion Molecule }\end{array}$ & $\begin{array}{c}\text { CD9 } \\
\text { CD29 } \\
\text { CD49d } \\
\text { CD54 (ICAM-1) } \\
\text { CD105 } \\
\text { CD106 (VCAM) } \\
\text { CD166 (ALCAM) }\end{array}$ \\
\hline Receptor molecules & $\begin{array}{l}\text { Hyaluronate receptors } \\
\text { Transferrin receptors }\end{array}$ & $\begin{array}{l}\text { CD44 } \\
\text { CD71 }\end{array}$ \\
\hline Surface enzymes & $\begin{array}{c}\text { Endopeptidase (Common Acute Lymphoblastic Leukemia Antigen, } \\
\text { Aminopeptidase } \\
\text { Ecto 5' nucleotidase }\end{array}$ & $\begin{array}{l}\text { CD10 (CALLA) } \\
\text { CD13 } \\
\text { CD73 }\end{array}$ \\
\hline $\begin{array}{l}\text { Extracellular matrix proteins } \\
\text { and glycoproteins }\end{array}$ & $\begin{array}{l}\text { Collagen I } \\
\text { Collagen III } \\
\text { Osteopontin } \\
\text { Osteonectin } \\
\text { Thy-1 } \\
\text { MUC-18 }\end{array}$ & $\begin{array}{c}- \\
- \\
- \\
- \\
\text { CD90 } \\
\text { CD146 }\end{array}$ \\
\hline Skeletal proteins & $\begin{array}{l}\text { Intracellular alpha smooth muscle actin (subset of all cells) } \\
\text { Vimentin }\end{array}$ & - \\
\hline $\begin{array}{l}\text { Complement regulatory pro- } \\
\text { teins }\end{array}$ & $\begin{array}{l}\text { Decay Accelerating Factor } \\
\text { Complement Protectin }\end{array}$ & $\begin{array}{l}\text { CD55 } \\
\text { CD59 }\end{array}$ \\
\hline Histocompatibility & A,B,C (Class I) & - \\
\hline
\end{tabular}


Table 2. Current Data on Adipose Tissue Derived Stem Cells Secretome

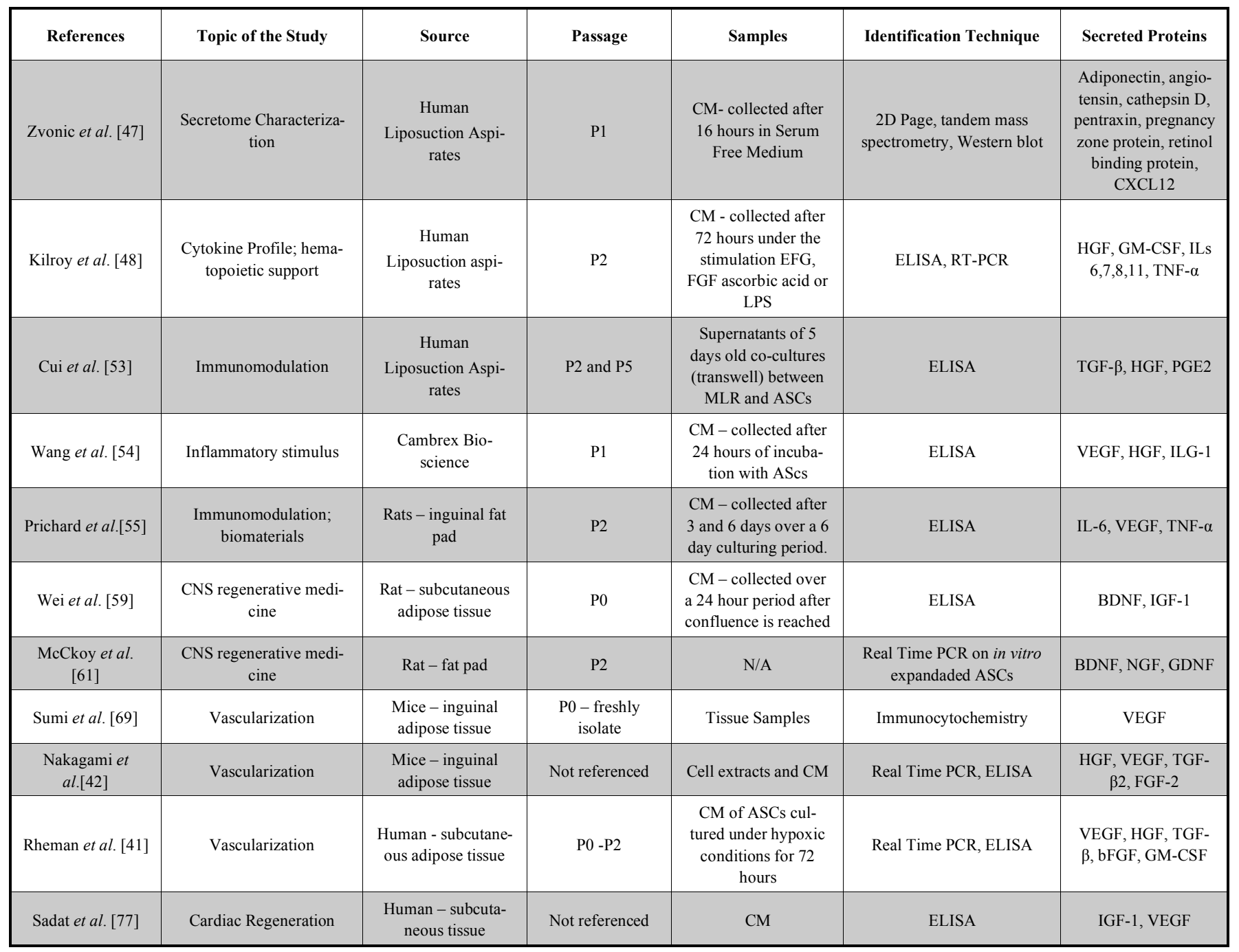

tent with secretion via the endoplasmatic reticulum/golgi dependent pathway is of particular interest. Within these $29 \%$, there were present multiple adipokines such as adiponectin, angiotensin, cathepsin $\mathrm{D}$, pentraxin, pregnancy zone protein and retinol binding protein. Moreover, leptin was also found, although in this case, only through antibody detection methods. Additionally molecules such as stromal cell-derived growth factor (CXCL12) were also identified.

Although the objectives of the second study were different, the paper by Kilroy et al. [48] provided complementary data on this topic. In this particular work the objective was to determine the cytokine profile of ASCs when exposed to different stimuli. Noteworthy to mention is the fact that none of the factors addressed in the manuscript were described in the 2D electrophoresis and MS study performed by Zvonic et al. [47]. Although intriguing, these differences could be related to the structural properties of the protein factors, which may limit the sensitivity of their detection through MS based techniques [47]. This study revealed that ASCs secrete different cytokines, such as hepatocyte growth factor (HGF), granulocyte and macrophage colony stimulating factor (GM-CSF), interleukins (ILs) 6, 7, 8 and 11 and tumor necrosis factor- $\alpha(\mathrm{TNF}-\alpha)$. Moreover, it was also shown that their release profile can be modulated following exposure to different agents. For instance, ASCs exposed to basic fibroblast growth factor (bFGF) or epidermal growth factor (EGF) significantly increased their release of HGF, a cytokine with roles in both hematopoiesis
[49] and vasculogenesis [50]. The HGF profile was further increased when ascorbic acid was present. These results relating to HGF are consistent with those of Rehman et al. [41] and Nakagami et al. [42] regarding the ability of ASCs to secrete angiogenic cytokines. In contrast, Kilroy et al. [48] also observed that ASCs could also respond to an inflammatory stimulus, such as lipopolysaccharide (LPS). Under these conditions, the ASCs increased their secretion of both hematopoietic (GM-CSF and IL-7) and proinflammatory (ILs 6, 8, 11 and TNF- $\alpha$ ) cytokines. These results confirm that ASCs display a cytokine secretory profile similar to that of BMSCs.

Both of these studies provided useful information on the ASCs secretome. However, other studies that do not specifically address release profile or the secretome, but rather the possible application of ASCs in regenerative medicine do also possess relevant content for this topic. Therefore, the following section will describe the relationship between these and other factors with the therapeutic potential of ASCs.

\section{MODULATION OF THE IMMUNE SYSTEM}

Cell based therapies, including ASCs, in combination with biomaterial and growth factor based approaches, play an important role on regenerative medicine strategies under development. It is therefore plausible to accept that once a few of these strategies are ready to be used in the current clinical practice there will be a de- 
mand for a reliable, safe and effective source of stem cells. Moreover, large numbers of cells will need to be obtained from these sources in order to facilitate a rapid application in the clinical theatre. In this sense the use of allogeneic populations, in addition to autologous cells, would be a major advantage from both the patient and commercial standpoints. By employing allogeneic cells, master cell banks could be created, where stem cells would be available whenever needed. In fact ASCs have recently shown the potential not only to be used in either autologous or allogeneic cell transplants, but simultaneously to act as modulators of the immune system [51-57].

For instance McIntosh and colleagues revealed that the frequency of hematopoietic-associated markers and histocompatible locus antigen-DR [HLA-DR] tend to decrease with subsequent passages of human ASCs. Additionally in mixed lymphocyte reactions (MLR), ASCs beyond P1 failed to elicit a response by MLR, suggesting the possible allogeneic potential of these cells. These in vitro results were then further confirmed by in vivo experiment in a spinal fusion model [52]. In this case results revealed that no $\mathrm{T}$ cell response was found and there was a low-titered, non-cytotoxic humoral response to the allogenic implants.

The work of Cui et al. [53] revealed some of the mechanisms associated to human ASCs mediated immunomodulatory effects. In this study it was shown that ASCs expressed HLA class I, but not class II molecules, which could only be induced upon ASCs incubation with interferon- $\gamma$ (IFN $\gamma$ ). Similarly to what had already been described in other reports, passaged ASCs did not stimulate lymphocyte proliferation. Additionally it was also found that either IFN $\gamma$ treated or non-treated ASCs could inhibit phytohemagglutinin (PHA)-stimulated lymphocyte proliferation. One of the striking findings in this manuscript was the evidence revealing that this immunosuppressive effect was not cell-to-cell contact dependent and therefore could be possibly attributed to soluble factors produced by ASCs. In order to determine if the suppressive effect was mediated by secreted cytokines, a cytokine profile was performed in conditioned media (CM) for the following molecules: Transforming Growth Factor- $\beta$ (TGF- $\beta$ ), HGF, prostaglandin E2 (PGE2) and IL-10. From these only the first three were detected in the CM through ELISA. Afterwards the level of each cytokine was measured in co-culture of ASCs with MLRs using a transwell culture system. Subsequent results revealed that of the three cytokines initially detected, only PGE2 levels were increased, suggesting that it might have a role in controlling the MLR proliferation. This hypothesis was then confirmed by measurement of an increased MLR proliterative response when an inhibitor of PGE2, indomethacin, was present. This important study provides some hints regarding the molecular and growth factor cues that regulate the immunomodulatory character of ASCs.

Wang et al. [54] also provided some important data regarding alterations of soluble factors produced by human ASCs following an inflammatory stimulus. In this case ASCs were incubated with TNF- $\alpha$ for $24 \mathrm{~h}$, after which their supernatants were screened for vascular endothelial growth factor (VEGF), HGF and insulin like growth factor 1 (IGF-1). Results revealed that all three of these soluble factors were significantly increased in response to the inflammatory stimuli.

Another interesting work is the one reported by Prichard et al. [55], which revealed that rat ASCs adhered to a polyurethane implant modulate, and in part favoured, the foreign body response to the latter in a rat model. Quite interesting from the perspective of the present review the screenings made on the supernatants of ASCs when seeded in either polystyrene or polyurethane implants. According to the results that were presented, the ASCs secretion of IL-6, VEGF and TNF- $\alpha$ could also be modulated by the surface upon which they were seeded. Thus, the bioscaffold/environment might be another mechanism to control the immunomodulatory character of ASCs.

\section{REGENERATIVE MEDICINE}

\subsection{Central Nervous System}

As a result of the limited capacity of the CNS to self-repair, neurodegenerative disorders and CNS related injuries (such as Parkinson's Disease (PD), Alzheimer's Disease, Spinal Cord Injuries and stroke/ischemia) have become one of the possible target applications for stem cell based therapies. The possible validity of ASCs for CNS regeneration purposes has been demonstrated in different models of injury and degeneration. These beneficial actions were initially attributed to the possible differentiation of ASCs towards the neuronal lineage. However, despite all the effort on this topic in recent years, there is no conclusive data yet on the in vivo differentiation of functional neurons from ASCs. Therefore, the strongest hypothesis to explain the role of ASCs in regeneration is mainly related to their possible trophic support provided through their secretome on the host cells $[58,59]$.

This hypothesis was initially tested using in vitro models. In order to determine the role of ASCs' soluble factors on neurospheres, Kang and colleagues [60] exposed the latter to human ASCs CM or, alternatively, co-cultured them using a cell culture transwell system. These experiments revealed that the neuronal differentiation of the neurospheres in the presence of conditioned medium was comparable to contact-dependent co-cultures, and thus indicated a possible role for soluble, as opposed to cell surface, factors. However, no information regarding which factors could be involved was provided by the authors. Nevertheless this work served as a background for forthcoming papers dealing with this subject. The reports of McCoy et al. [61] and Wei et al. [59] are good examples of this.

In the first paper the goal was to study the role of nondifferentiated and differentiated ASCs on a rat model of PD. One week after the injury, differentiated and non-differentiated ASCs were injected in the substantia nigra. Neurochemical and behavioral analyses indicated that both populations abrogated dopaminergic neuronal cell death, which suggested that the pre-differentiation of ASCs towards the neuronal pathway did not determine the ability of ASCs to survive or protect the host populations in vivo. Moreover, immunohistological analysis failed to show robust differentiation of both cell transplants, a fact that further reinforces the view that the beneficial effects of ASCs in the CNS display trophic characteristics. In addition to this, the authors also made an extensive gene expression analysis on possible soluble factors that could be implicated in the observed phenomena. Quantitative real time PCR and semi-quantitative RT-PCR experiments detected high levels of Brain Derived Neurotrophic Factor (BDNF), Nerve Growth Factor (NGF) and Glial Derived Neurotrophic Factor (GDNF). The first two are well known growth factors involved in both the differentiation of new neurons and the protection/survival of established differentiated cells $[62,63]$. In contrast, GDNF is particularly involved in the specific survival and differentiation of dopaminergic neurons $[64,65]$. Moreover, the expression of the genes encoding these factors was potentiated whenever EFG/FGF-2 were present in the medium. Although one must recall that this was a gene expression analysis, and not the quantification of the factor itself, the results presented in this report suggest that the ASC secretome will play a role in future CNS regenerative medicine studies.

The paper by Wei and colleagues [59] further explores the CNS potential of the rat ASCs secretome. In this particular report only $\mathrm{CM}$, rather than cells, were used. The objective was to study the neuroprotective effect of $\mathrm{CM}$ in a rat model of brain hypoxicischaemic (HI) injury. Concentrated CM from cultured rat ASCs (ASC-CM) or control medium were infused through the jugular vein of neonatal Sprague-Dawley rats subjected to HI injury. The ASC-CM was administered either 1 hour before or 24 hours after induction of injury. Analysis at 1 week indicated that administration at both time points significantly protected against hippocampal and 
cortical volume loss. Analysis of parallel groups for behavioral and learning changes at 2 months post-ischemia demonstrated that both treated groups performed significantly better than the controls in Morris water maze functional tests. Particularly interesting was the confirmation of penetration of the blood brain barrier by protein components of the injected CM, as well as their predominant binding to structures within affected regions of the brain. Additionally the authors attribute these findings to IGF-1 and BDNF. The first apparently provides protection against apoptosis, while the second can be involved, for instance, in the protection of glutamate excitotoxicity. However, it should be said that these two factors do not necessarily account for all the observed phenomena, and thus a more in depth characterization on the neurotrophic factors present in the media is mandatory. Together, these publications demonstrate that identified growth factors within the ASC secretome can positively modulate recovery and regeneration within the injured CNS.

\subsection{Vascularization}

Angiogenesis, which is the process that leads to the formation of new blood vessels, is a key event in any process of regenerative medicine. Through the years the concept of therapeutic angiogenesis to target problems such as tissue ischemia or to accelerate wound healing has emerged as one of them most promising therapies to date [66]. Its concept is based on the stimulation of the proliferation of collateral blood vessels that ultimately will lead to the re-vascularization of the affected area. Early clinical trials reported that the administration of angiogenic growth factors could enhance the formation of such vessels [67]. However the clinical efficacy of administering a single factor is still quite controversial $[68,69]$. As it happened with other areas of regenerative medicine ASCs and their secretome have been involved in a series of novel methodologies that are able to enhance angiogenesis in different conditions. Nearly simultaneously, pioneering studies by Planat-Bernard [34], Rehman [41], Miranville [70] and colleagues demonstrated that human ASCs displayed endothelial-like functionality in vitro and in vivo using hind limb ischemia models.

More recently, Sumi and colleagues [69] reported the use of murine ASCs in an ischemic limb mouse model. The latter was achieved by resecting the right femoral and saphenous artery. ASCs were injected on the same day, using bone marrow MSCs, mature adipocytes and PBS as controls. Results revealed that ASCs significantly augmented collateral blood vessel development that allowed the restoration of blood perfusion and capillary density in the ischemic model. These results were quite similar to those obtained for bone marrow MSCs, which are commonly viewed as the gold standard regarding mesenchymal like stem cells populations. Although it was also observed that a small number of ASCs differentiated into endothelial and smooth muscle cells, the authors attributed most of the success to the expression of VEGF, as assessed by immunocytochemistry. However, it may be short sighted to attribute the response solely to VEGF since ASCs do express a myriad of growth factors, some of which are endowed with angiogenic properties.

Another study on this topic by Nakagami et al. [42] shed light on the possible mechanisms how soluble factors expressed by murine ASCs can induce angiogenesis. Their in vitro real time PCR studies showed that besides HGF, VEGF, TGF- 32 and FGF-2, already mentioned in this manuscript, ASCs also expressed the genes for placental growth factor (PGF) and angiopoietins 1 and 2 (Ang-1 and Ang-2). The expression of HGF and VEGF was then further confirmed by ELISA, with the concentration of HGF being higher than that of VEGF. Moreover the secretion of these two factors could be manipulated by adding different supplements to the culture system, a fact indicating that the action of ASCs in vivo could be determined by the local micro-environments. Finally their role on endothelial cells (ECs) viability and proliferation was assessed by treating ASCs CM with neutralizing antibodies for HGF and VEGF. With anti-HGF antibodies, there was a $25 \%$ inhibition on cell viability and a $48 \%$ inhibition on ECs migration, while antiVEGF antibodies led to an inhibition of around $23 \%$ on cell viability and $26 \%$ on cell migration. Under these conditions it seems that although both factors have a similar role on maintaining cell viability, HGF has a striking effect on ECs migration. Therefore HGF secreted by ASCs seems to display a crucial role in recruiting ECs in vivo for the formation of new blood vessels.

The importance and role of HGF secreted by human ASCs on the angiogenesis of ischemic tissue was further demonstrated by Cai et al. [71]. In this work the expression of HGF was reduced to about $80 \%$ of the normal level by silencing HGF expression through RNA interference. In vitro experiments revealed that CM from these "HGF impaired" ASCs had a reduced ability to promote survival, proliferation and migration of both mature and progenitor endothelial cells. Moreover it was also observed that when compared to control populations of ASCs, the HGF silenced cell populations had also a reduced ability for promoting reperfusion in a mouse ischemic hind-limb model. Additionally it was also seen that in these cases there were smaller densities of capillaries and fewer ASCs in the ischemic limb.

Another interesting report regarding the beneficial effects of the soluble factors secreted by human ASCs was put forward by Rehman et al. [41]. In this work it was shown that when cultured in hypoxic conditions ASCs were able to increase their VEGF secretion by 5 fold. Moreover, CM obtained from ASCs grown under hypoxic condition were able to increase the growth of endothelial cells and simultaneously to reduce the apoptosis rate of this population of cells. These experiments were a clear indicator of the role of the surrounding environment on ASCs secretome. Moreover, the way that these cells reacted to hypoxic conditions is a further indicator on their possible role in clinical conditions that involve ischemia.

Overall these reports clearly show the importance of the secretome, namely HGF and VEGF, of ASCs in inducing angiogenesis in areas that have undergone ischemic episodes. However, it is not only in ischemic tissues that vascularization is important. In processes such as wound healing the formation of new blood vessels is also extremely important, and ASCs have potential utility in such models. Kim et al. [72] have shown the role of different cytokines in controlling fibroblast proliferation/migration, which could obviously affect the time required for wounds to heal. Moreover, they have [73] also shown that ASCs could protect fibroblasts from oxidative stress. Although the authors did not attribute these results to a specific factor, the paper refers to a proteomic based analysis of ASCs CM that reveal the presence of different soluble factors that might be involved in this process. Given this, Blanton and colleagues [74] used a porcine full-thickness wound model to evaluate the therapeutic usage of ASCs for these purposes. Results revealed that although there were no differences in re-epithelization, the groups treated with ASCs had higher densities of microvessels. Simultaneously the ASCs were found near the new vasculature and the VEGF levels were higher in the groups using ASCs, thus supporting the importance of these cells in regulating wound repair.

\subsection{Cardiac Regeneration}

Cardiac regeneration has been another area where ASCs have been applied and the effects of their secretome have been observed. In fact, there have been a number of papers reporting positive effects of these cells when transplanted/injected in different animal models of myocardial infarction/failure $[75,76]$. Similar to other ASC applications in ischemic models, the improvement on the cardiac regeneration in these animal models was attributed to an increase in vascularization and/or a cell protective effect, namely by reducing apoptosis. Although the information is limited, Sadat and colleagues [77] identified the contribution of VEGF and IGF-1 to 
this phenomenon in co-culture studies using human ASCs. Through RNA silencing experiments it was possible to conclude that $62.5 \%$ of this anti-apoptotic effect was mediated by IGF, while, as expected, VEGF was mainly involved in the promotion of angiogenesis. Thus, the angiogenic and anti-apoptotic actions of the ASC secretome contribute to tissue repair in multiple depots by preventing post-ischemic damage. In addition to the growth factors mentioned, it is likely that other ASC derived proteins contribute to this favourable outcome; however, additional studies will be necessary to validate this speculation.

\section{CONCLUSIONS}

Multiple studies demonstrate that ASCs secrete several soluble factors with effects on different cell populations. At the immune system level there is substantial evidence that PGE2 partially regulates some of the immunomodulatory properties/effects of ASCs. Moreover, ASCs also respond to inflammatory stimuli from their environment by increasing their expression of angiogenic factors such as VEGF, HGF and IGF-1 as well as hematopoietic/inflammatory factors such as G-CSF, M-CSF, IL-6 and TNF- $\alpha$. Regarding the CNS, the factors implicated are different and it is currently known that BDNF, GDNF, NGF and IGF secreted by ASCs have different roles on neuroprotection and differentiation. Finally there is evidence suggesting that IFG-1 secreted by ASCs may protect cardiomyocytes from apoptotic cell death. Overall, it is evident that many ASC secreted factors act through mechanisms that mediate protection against cell death or, alternatively, induce cell migration and proliferation. Alternatively they can indirectly act on the targeted cell populations. For instance by promoting vascularization, they can be indirectly linked to an increase of oxygen and nutrients in the affected areas, which may than promote local regenerative processes. Although this information is quite useful, given the plethora of factors that are expressed by ASCs, it is expected that other factors might affect, in different forms, fully differentiated and progenitor cells at the target tissues. It will be then necessary to employ more powerful techniques. Up to now, most of the reports have focused on the same factors. While this is useful as it allows us to understand the different function of this small set of growth factors, it limits discovery approaches towards the secretome as a therapeutic tool. Therefore the application of unbiased, global discovery techniques such as liquid chromatography/MS need to be applied and the resulting data correlated with that obtained by complementary approaches such as, for instance, ELISA or luminex based assays. By doing so, a better understanding of the secretome will be attained, with the possibility of discovering new potential factors. In addition to this information it is advisable that serial studies be made on how the secretome changes according to the passage of cells, as the latter will be most likely different from passage to passage. Additionally other questions can also be posed. For instance it remains unclear if it is preferable to transplant ASCs or, alternatively, their CM; both approaches may have a therapeutic potential best suited to a particular pathology dependent on the target disease/injury. If a prolonged release of factors is needed, then ASCs transplant may be beneficial. The use of genetically or culture condition modified ASCs capable of releasing induced levels of a particular cytokine or growth factor merits consideration. Alternatively, if an initial burst of factors is desired, than the injection of concentrated CM from ASCs would be the most logical approach. Another relevant topic is how the secretome of ASCs can be modulated. A possible hypothesis could be through the use of bioreactors, as ASCs are sensitive to dynamic environments. Another possibility would be the use of defined media that specifically trigger the expression of the desired trophic factors. Alternatively the growth of these cells in suspension based bioreactor systems and in contact with engineered biomaterials surfaces, could also be possible routes to follow. With these approaches, it will probably be possible to expand the applications of ASCs, and their secretome, to a wider range of regenerative medicine protocols in a rational, evidence-based manner.

\section{REFERENCES}

[1] Schäffler A, Büchler C. Adipose tissue-derived stromal cells basic and clinical applications for novel cell therapies. Stem Cells 2007; 25: 818-27.

[2] Kershaw EE, Flier JS. Adipose tissue as an endocrine organ. J Clin Metab 2004; 89(6): 2548-56.

[3] Spalding KL, Arner E, Westermark PO, et al. Dynamics of fat cell turnover in humans. Nature 2008; 453(7196): 783-7.

[4] Gimble JM, Katz AJ, Bunnell BA. Adipose-tissue derived stem cells for regenerative medicine. Circ Res 2007; 100: 1249-60.

[5] Safford KM, Rice HE. Stem cell therapy for neurologic disorders: Therapeutic potential of adipose-derived stem cells. Cur Drug Targets 2005; 6: 57-62.

[6] Kaplan FS, Hahn GV, Zasloff MA. Heterotopic ossification: two rare forms and what they can teach us. J Am Acad Orthop Surg 1994; 2: 288-96.

[7] Eddy MC, Jan de Beur SM, Yandow SM, et al. Deficiency of the alpha- subunit of the stimulatory g protein and severe extraskeletal ossification. J Bone Miner Res 2000; 15: 2074-83.

[8] Yeh GL, Mathur S, Wivel A, et al. Gnas1 mutation and cbaf1 misex-pression in a child severe congenital platelike osteoma cutis. J Bone Miner Res 2000; 15: 2063-73.

[9] Cornelius P, MacDougald OA, Lane MD. Regulation of adipocyte development. Ann Rev Nutr 1994; 14: 99-129.

[10] Neese RA, Mesell LM, Turner S, et al. Measurement in vivo proliferation rates slows down turnover cell by $2 \mathrm{~h} 2 \mathrm{o}$ labeling of deoxyribose moiety of DNA. Proc Natl Acad Sci USA 2002; 99: 1534550 .

[11] Strawford A, Antelo F, Christiansen M, Hellerstein MK. Adipose tissue triglyceride turnover, de novo lipogenesis, and cell proliferation in human measured with 2h2o. Am J Physiol Endocrinol Metab 2004; 286: E577-E588.

[12] Björntorp P, Karlsson M, Pertoft H, Pettersson P, Sjöström L Smith U. Isolation and characterization of cells from rat adipose tissue developing into adipocytes. J Lipid Res 1978; 19(3): 316-24.

[13] Hauner H, Entenmann G, Wabitsch M, Gaillard D, et al. Promoting effect of glucocorticoids on the differentiation of human adipocyte precursor cells cultured in a chemically defined medium. J Clin Invest 1989; 84(5): 1663-70.

[14] Van RL, Bayliss CE, Roncari DA. Cytological and enzymological characterization of adult human adipocyte precursors in culture. $\mathrm{J}$ Clin Invest 1976; 58(3): 699-704.

[15] Halvorsen YC, Wilkison WO, Gimble JM. Adipose-derived stromal cells--their utility and potential in bone formation. Int $\mathrm{J}$ Obes Relat Metab Disord 2000; 24 (Suppl 4): S41-S44.

[16] Zuk PA, Zhu M, Mizuno H, et al. Multilineage cells from human adipose tissue: Implications for cell-based therapies. Tissue Eng 2001; 7(2): 211-28.

[17] Erickson GR, Gimble JM, Franklin DM, Rice HE, Awad H, Guilak F.Chondrogenic potential of adipose tissue-derived stromal cells in vitro and in vivo. Biochem Biophys Res Commun 2002; 290(2): 763-9.

[18] Rada T, Reis RL, Gomes ME. Adipose tissue-derived stem Cells and their application in bone and cartilage tissue engineering. Tissue Eng Part B Rev 2009; [Epub ahead of print].

[19] Gimble JM, Guilak F. Adipose-derived adult stem cells: Isolation, characterization, and differentiation potential. Cytotherapy 2003; 5(5): 362-9.

[20] Hauner H, Entenmann G, Wabitsch M, et al. Promoting effect of glucocorticoids on the differentiation of human adipocyte precursor cells cultured in a chemically defined medium. J Clin Invest 1989; 84(5): 1663-70.

[21] Deslex S, Negrel F, Vannier C, et al. Differentiation of human adipocyte precursors in a chemically defined serum-free medium. Int J Obes 1986; 10: 19-27

[22] Rodriguez AM, Elabd C, Amri E-Z, Ailhaud G, Dani C. The human adipose tissue is a source of multipotent stem cells. Biochimie 2005; 87: 125 . 
[23] Baksh D, Song L, Tuan RS. Adult mesenchymal stem cells: Characterization, differentiation, and application in cell and gene therapy. J Cell Mol Med 2004; 8: 301-16.

[24] Salgado AJ, Oliveira JT, Pedro AJ, Reis RL. Adult stem cells in bone and cartilage tissue engineering. Curr Stem Cell Res Ther 2006; 1(3): 345-64.

[25] Hicok KC, Du Laney TV, Zhou YS, et al. Human adipose-derived adult stem cells produce osteoid in vivo. Tissue Eng 2004; 10: 371 80.

[26] Awad HA, Wickham MQ, Leddy HA, Gimble JM, Guilak F. Chondrogenic differentiation of adipose-derived adult stem cells in agarose, alginate, and gelatin scaffolds. Biomaterials 2004; 25 : 3211-22

[27] Betre H, Ong SR, Guilak F, et al. Chondrocytic differentiation of human adipose-derived adult stem cells in elastin-like polypeptide. Biomaterials 2006; 27: 91-9.

[28] Kim YM, Jeon ES, Kim MR, Jho SK, Ryu SW, Kim JH. Angiotensin II-induced differentiation of adipose tissue-derived mesenchymal stem cells to smooth muscle-like cells. Int J Biochem Cell Biol 2008; 40(11): 2482-91.

[29] Safford KM, Hicok KC, Safford SD, et al. Neurogenic differentiation of murine and human adipose-derived stromal cells. Biochem Biophys Res Commun 2002; 294(2): 371-9.

[30] Safford KM, Safford SD, Gimble JM, Shetty AK, Rice E. Characterization of neuronal/glial differentiation of murine adipose-derived adult stromal cells. Exp Neurol 2004; 187(2): 319-28.

[31] van Dijk A, Niessen HW, Zandieh Doulabi B, Visser FC, van Milligen FJ. Differentiation of human adipose-derived stem cells towards cardiomyocytes is facilitated by laminin. Cell Tissue Res 2008; 334(3): 457-67.

[32] Zhu Y, Liu T, Song K, Ning R, Ma X, Cui Z. ADSCs differentiated into cardiomyocytes in cardiac microenvironment. Mol Cell Biochem 2009; 324(1-2): 117-29

[33] Cao Y, Sun Z, Liao L, Meng Y, Han Q, Zhao RC. Human adipose tissue-derived stem cells differentiate into endothelial cells in vitro and improve postnatal neovascularization in vivo. Biochem Biophys Res Commun 2005; 332(2): 370-9.

[34] Planat-Benard V, Silvestre JS, Cousin B, et al. Plasticity of human adipose lineage cells toward endothelial cells: physiological and therapeutic perspectives. Circulation 2004; 109(5): 656-63.

[35] Brzoska M, Geiger H, Gauer S, Baer P. Epithelial differentiation of human adipose tissue-derived adult stem cells. Biochem Biophys Res Commun 2005; 330(1): 142-50.

[36] Baer PC, Bereiter-Hahn J, Missler C, et al. Conditioned medium from renal tubular epithelial cells initiates differentiation of human mesenchymal stem cells. Cell Prolif 2009; 42(1): 29-37.

[37] Chen X, Cushman SW, Pannell LK, Hess S. Quantitative proteomic analysis of the secretory proteins from rat adipose cells using a 2D liquid chromatography-MS/MS approach. J Proteome Res 2005; 4(2): 570-7.

[38] Wang P, Mariman E, Keijer J, et al. Profiling of the secreted proteins during 3T3-L1 adipocyte differentiation leads to the identification of novel adipokines. Cell Mol Life Sci 2004; 61(18): 240517.

[39] Kratchmarova I, Kalume DE, Blagoev B, et al. A proteomic approach for identification of secreted proteins during the differentiation of 3T3-L1 preadipocytes to adipocytes. Mol Cell Proteomics 2002; 1(3): 213-22.

[40] Choi KL, Wang Y, Tse CA, Lam KS, Cooper GJ, Xu A. Proteomic analysis of adipocyte differentiation: Evidence that alpha2 macroglobulin is involved in the adipose conversion of 3T3 L1 preadipocytes. Proteomics 2004; 4(6): 1840-8.

[41] Rehman J, Traktuev D, Li J, et al. Secretion of angiogenic and antiapoptotic factors by human adipose stromal cells. Circulation 2004; 109(10): 1292-8.

[42] Nakagami H, Maeda K, Morishita R, et al. Novel autologous cell therapy in ischemic limb disease through growth factor secretion by cultured adipose tissue-derived stromal cells. Arterioscler Thromb Vasc Biol 2005; 25(12): 2542-47.

[43] DeLany JP, Floyd ZE, Zvonic S, et al. Proteomic analysis of primary cultures of human adipose-derived stem cells: modulation by Adipogenesis. Mol Cell Proteomics 2005; 4(6): 731-40.
[44] Molina H, Yang Y, Ruch T, et al. Temporal profiling of the adipocyte proteome during differentiation using a five-plex SILAC based strategy. J Proteome Res 2009; 8(1): 48-58.

[45] Hausman GJ, Poulos SP, Richardson RL, et al. Secreted proteins and genes in fetal and neonatal pig adipose tissue and stromalvascular cells. J Anim Sci 2006; 84(7): 1666-81.

[46] Chiellini C, Cochet O, Negroni L, et al. Characterization of human mesenchymal stem cell secretome at early steps of adipocyte and osteoblast differentiation. BMC Mol Biol 2008; 9: 26

[47] Zvonic S, Lefevre M, Kilroy G, et al. Secretome of primary cultures of human adipose-derived stem cells: modulation of serpins by adipogenesis. Mol Cell Proteomics 2007; 6(1): 18-28.

[48] Kilroy GE, Foster SJ, Wu X, et al. Cytokine profile of human adipose-derived stem cells: expression of angiogenic, hematopoietic, and pro-inflammatory factors. J Cell Physiol 2007; 212(3): 702-9.

[49] Sugiura K, Taketani S, Yoshimura T, et al. Effect of hepatocyte growth factor on long term hematopoiesis of human progenitor cells in transgenic-severe combined immunodeficiency mice. Cytokine 2007 ; 37(3): 218-26.

[50] Aoki M, Morishita R, Taniyama Y, et al. Angiogenesis induced by hepatocyte growth factor in non-infarcted myocardium and infarcted myocardium: up-regulation of essential transcription factor for angiogenesis. Gene Ther 2000; 7(5): 417-27.

[51] McIntosh K, Zvonic S, Garrett S, et al. The immunogenicity of human adipose-derived cells: temporal changes in vitro. Stem Cells 2006; 24(5): 1246-53.

[52] McIntosh KR, Lopez MJ, Borneman JN, Spencer ND, Anderson PA, Gimble JM. Immunogenicity of allogeneic adipose-derived stem cells in a rat spinal fusion model. Tissue Eng Part A 2009, in press.

[53] Cui L, Yin S, Liu W, Li N, Zhang W, Cao Y. Expanded adiposederived stem cells suppress mixed lymphocyte reaction by secretion of prostaglandin E2. Tissue Eng 2007; 13(6): 1185-95.

[54] Wang M, Crisostomo PR, Herring C, Meldrum KK, Meldrum DR. Human progenitor cells from bone marrow or adipose tissue produce VEGF, HGF, and IGF-I in response to TNF by a p38 MAPKdependent mechanism. Am J Physiol Regul Integr Comp Physiol 2006; 291(4): R880-4.

[55] Prichard HL, Reichert W, Klitzman B. Adipose-derived stromal cells improve the foreign body response. Stem Cells 2008; 26(10): 2691-95.

[56] Niemeyer P, Kornacker M, Mehlhorn A, et al. Comparison of immunological properties of bone marrow stromal cells and adipose tissue-derived stem cells before and after osteogenic differentiation in vitro. Tissue Eng 2007; 13(1): 111-21.

[57] Puissant B, Barreau C, Bourin P, et al. Immunomodulatory effect of human adipose tissue-derived adult stem cells: comparison with bone marrow mesenchymal stem cells. Br J Haematol 2005; 129(1): 118-29.

[58] Jin K, Greenberg DA. Tales of differentiation. Exp Neurol 2003; 183: 255-7.

[59] Wei X, Du Z, Zhao L, et al. The conditioned media of adipose Stromal cells protect against hypoxia-ischemia-induced brain damage in neonatal rats. Stem Cells 2009; 27(2): 478-88.

[60] Kang SK, Jun ES, bae YC, Jung JS. Interaction between human adipose stromal cells and mouse neural stem cells. Dev Brain Res 2003; 145: 141-9.

[61] McCoy MK, Martinez TN, Ruhn KA, et al. Autologous transplants of Adipose-Derived Adult Stromal (ADAS) cells afford dopaminergic neuroprotection in a model of Parkinson's disease. Exp Neurol 2008; 210 (1): 14-29.

[62] Li T, Jiang L, Zhang X, Chen H. In-vitro effects of brain-derived neurotrophic factor on neural progenitor/stem cells from rat hippocampus. Neuroreport 2009; 20(3): 295-300.

[63] Gu H, Long D, Song C, Li X. Recombinant human NGF-loaded microspheres promote survival of basal forebrain cholinergic neurons and improve memory impairments of spatial learning in the rat model of Alzheimer's disease with fimbria-fornix lesion. Neurosci Lett 2009; 453(3): 204-9.

[64] Evans JR, Barker RA. Neurotrophic factors as a therapeutic target for Parkinson's disease. Expert Opin Ther Targets 2008; 12(4): 437-47. 
[65] Sandhu JK, Gardaneh M, Iwasiow R, et al. Astrocyte-secreted GDNF and glutathione antioxidant system protect neurons against 6-OHDA cytotoxicity. Neurobiol Dis 2009; 33(3): 405-14.

[66] Freedman SB, Isner JM. Therapeutic angiogenesis for ischemic cardiovascular disease. J Mol Cell Cardiol 2001; 33(3): 379-93.

[67] Henry TD, Annex BH, McKendall GR, et al. The VIVA trial: Vascular endothelial growth factor in Ischemia for Vascular Angiogenesis. Circulation 2003; 107(10): 59-1365.

[68] Kastrup J, Jørgensen E, Rück A, et al. Direct intramyocardial plasmid vascular endothelial growth factor-A165 gene therapy in patients with stable severe angina pectoris A randomized doubleblind placebo-controlled study: The Euroinject One trial. J Am Coll Cardiol 2005; 45(7): 982-8.

[69] Sumi M, Sata M, Toya N, Yanaga K, Ohki T, Nagai R. Transplantation of adipose stromal cells, but not mature adipocytes, augments ischemia-induced angiogenesis. Life Sci 2007; 80(6): $559-65$.

[70] Miranville A, Heeschen C, Sengenès C, Curat CA, Busse R, Bouloumié A. Improvement of postnatal neovascularization by human adipose tissue-derived stem cells. Circulation 2004; 110(3): 349-55.

[71] Cai L, Johnstone BH, Cook TG, et al. Suppression of hepatocyte growth factor production impairs the ability of adipose-derived stem cells to promote ischemic tissue revascularization. Stem Cells 2007; 25(12): 3234-43.

[72] Kim WS, Park BS, Sung JH, et al. Wound healing effect of adipose-derived stem cells: a critical role of secretory factors on human dermal fibroblasts. J Dermatol Sci 2007; 48(1): 15-24.

[73] Kim WS, Park BS, Kim HK, et al. Evidence supporting antioxidant action of adipose-derived stem cells: protection of human dermal fibroblasts from oxidative stress. J Dermatol Sci 2008; 49(2): 13342.

[74] Blanton MW, Hadad I, Johnstone BH, et al. Adipose stromal cells and platelet-rich plasma therapies synergistically increase revascularization during wound healing. Plast Reconstr Surg 2009; 123(2 Suppl): S56-64S.

[75] Cai L, Johnstone BH, Cook TG, et al. Human adipose tissuederived stem cells Induce angiogenesis and nerve sprouting following myocardial infarction, in conjunction with potent preservation of cardiac function. Stem Cells 2009; 27(1): 230-37.

[76] Schenke-Layland K, Strem BM, Jordan MC, et al. Adipose tissuederived cells improve cardiac function following myocardial infarction. J Surg Res 2009; 153(2): 217-23.

[77] Sadat S, Gehmert S, Song YH, et al. The cardioprotective effect of mesenchymal stem cells is mediated by IGF-I and VEGF. Biochem Biophys Res Commun 2007; 363 (3): 674-9. 